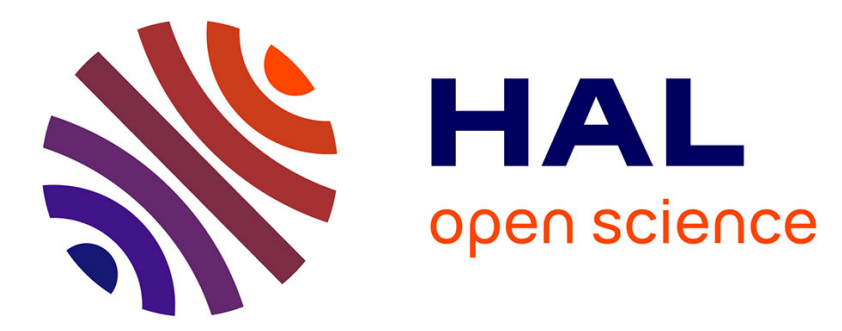

\title{
Experimental study of the instability of the Hartmann layer
}

\author{
Pablo Moresco, Thierry Alboussiere
}

\section{To cite this version:}

Pablo Moresco, Thierry Alboussiere. Experimental study of the instability of the Hartmann layer. Journal of Fluid Mechanics, 2003, 504, pp.167-181. 10.1017/S0022112004007992 . hal-00203508

\section{HAL Id: hal-00203508 \\ https://hal.science/hal-00203508}

Submitted on 10 Jan 2008

HAL is a multi-disciplinary open access archive for the deposit and dissemination of scientific research documents, whether they are published or not. The documents may come from teaching and research institutions in France or abroad, or from public or private research centers.
L'archive ouverte pluridisciplinaire HAL, est destinée au dépôt et à la diffusion de documents scientifiques de niveau recherche, publiés ou non, émanant des établissements d'enseignement et de recherche français ou étrangers, des laboratoires publics ou privés. 


\title{
Experimental study of the instability of the
}

\author{
Hartmann layer \\ By P. MORESCO†AND T. ALBOUSSIÉRE \\ Department of Engineering, University of Cambridge, Trumpington Street, Cambridge CB2 \\ 1PZ, UK.
}

Grenoble High Magnetic Field Laboratory, 25 avenue des Martyrs, BP 166 Grenoble Cedex 9,

France

(Received 27 May 2003)

Hartmann layers are a common feature in magnetohydrodynamics where they organise the electric current distribution in the flow and hence the characteristics of the velocity field. In spite of their importance their stability properties are not well understood, mainly because of the scarcity of experimental data. In this work we investigated experimentally the transition to turbulence in the Hartmann layers that arise in magnetohydrodynamic flows in ducts. From measurements of the friction factor a well marked transition to turbulence was found at a critical Reynolds number, based on the laminar Hartmann layer thickness, of approximately 380, valid also for laminarisation and for a wide range of intensities of the magnetic field. The sensitivity of this result to the roughness characteristics of the walls along which the Hartmann layers develop, confirms that these layers are related to the transition observed and provides more information on its stability properties.

$\dagger$ Present address: Department of Physics and Astronomy, The University of Manchester, Manchester M13 9PL, UK. 


\section{Introduction}

In magnetohydrodynamics (MHD), Hartmann layers arise along boundaries non-parallel to an imposed magnetic field. Because they act as a path for the electric currents they have a dominant effect on the velocity field in the core of the flow (Moreau 1990). Information on the parameter ranges for which the layer is laminar or turbulent is crucial in any activity where a detailed control of the velocity field is necessary, such as in the casting of metals and the growth of semiconductor crystals in the presence of a magnetic field (Garandet \& Alboussière 1999). In spite of its importance, there are no detailed experimental results on the transition to turbulence in Hartmann layers.

Since the invention in 1918 of the electromagnetic pump by Hartmann (1937), several studies (Hartmann \& Lazarus 1937; Murgatroyd 1953; Lykoudis 1960; Branover 1967) investigated how global properties of pressure driven MHD flows in ducts, such as their friction factors, are determined by the intensity of the externally imposed magnetic field. Hartmann \& Lazarus (1937) found that in the laminar regime an external transverse magnetic field induces changes in the velocity profile that result in an increase of the "apparent viscosity" and the pressure drop along the duct. They also found that in the turbulent regime this effect is counteracted by the damping of vorticity in the direction perpendicular to the magnetic field, which can result in a decrease of the pressure drop. Murgatroyd (1953) found that the friction factor in those flows is a function of the ratio of the Reynolds number $(R e)$ to the Hartmann number $(H a)$, which is equivalent to a Reynolds number $(R)$ based on the thickness of the Hartmann layer. These experiments focused on the transition from a turbulent to a laminar regime, and although they dealt with ducts of different shapes and aspect ratios, the critical value of $R$ for laminarisation was consistently found to be in the range of $150<R<250$.

At high Hartmann number the theory of MHD flows in ducts predicts that, apart 
from the Hartmann boundary layers, parallel or secondary layers will develop along the boundaries parallel to the magnetic field (Hunt 1965). The thickness of the parallel layers is $O\left(H a^{-1 / 2}\right)$ (figure 1 ) while that of the Hartmann layers is $O\left(H a^{-1}\right)$, and according to the linear stability theory they possess very different stability properties. The role that each boundary layer plays in the laminarisation and transition to turbulence processes in ducts is not so far well understood.

The stability properties of the parallel layers and the related heat transport across them were studied in electrically conducting ducts because of their relevance to the cooling of fusion blankets. In this configuration most of the electric current flows in the walls of the duct and Hunt (1965) showed that the local velocity in the parallel layers can locally exceed that in the core of the duct. Reed \& Picologlou (1989) measured the streamwise velocity component in the mid-plane of a rectangular duct at high Hartmann number $\left(O\left(10^{3}\right)\right)$, and found that instability first appears in the proximity of the wall parallel to the magnetic field in the range $2650<R e<5100$, independently of the value of the Hartmann number, but that the core of the flow remains laminar. Similar behaviour was found experimentally by Burr et al. (2000), who reported that for high magnetic fields the turbulence in the parallel layers takes the form of vortex tubes orientated parallel to the field, and that the pressure losses resulting from this two-dimensional turbulence are small compared to the losses originating from Joule's dissipation, which takes place mostly in the Hartmann layers. A linear stability analysis of the parallel layers by Ting \& Walker (1991) yielded a critical Reynolds number of 313, again independent of the value of $H a$. Besides there being an order of magnitude difference between this value and the experimental results cited above, probably due to nonlinear effects, both results are well below those found experimentally for laminarisation in ducts. This seems to indicate that the transition detected through global measurements in those MHD flows is not directly 
related to the conditions of the parallel layers. On the other hand, the relevance of the Hartmann layer thickness as the characteristic length in those transitions suggests that the stability of these layers may play a more important role. We are not aware of any experimental or theoretical study of the stability of the parallel layers where the walls non-parallel to the magnetic field (Hartmann walls) were insulating. In the later case most of the electric currents are restricted to the Hartmann layers and their intensity is linearly related to the velocity in the core of the flow.

A linear stability analysis of the Hartmann layer was carried out by Lock (1955) who found that, at high $\mathrm{Ha}$, the only effect of the Lorentz forces is in determining the basic velocity profile and that the critical Reynolds over Hartmann number for transition is approximately 50000. Lingwood \& Alboussière (1999) confirmed that this instability mode has a viscous origin, as the effect of the Lorentz forces on the stability equations is very small. The high value of the critical $R$ is then to be expected, as the unperturbed velocity profile is exponential and coincides with that of the asymptotic suction layers, known to be very stable (Schlichting 1960). Nonlinear effects may be responsible for the big difference with experimental results on laminarisation, making a comparison between transition and laminarisation particularly difficult. Moresco \& Alboussière (2002b) showed that the isolated Hartmann layer is unstable to perturbations of small but finite size for values of $R$ below and in the vicinity of the critical according to the linear analysis. This can lead to hysteresis in the transition process as the disturbances present in the turbulent Hartmann layer can make the flow unstable at subcritical values of the Reynolds number.

The main aim of this work is to show the relevance of the Hartmann layers in the observable transitions in MHD flows and to determine experimentally the critical value of $R$ for transition. In $\S 2$ we describe the experimental set-up and the measurements 
obtained. The results of the experiments showing the dependence of the friction factor with the intensity of the magnetic field and the velocity of the flow are presented and discussed in $\S 3$. The effect of roughness on the surface in contact with the Hartmann layers is investigated in the same section. The findings and their relation to analytical results are summarised in $\S 4$.

\section{Experimental arrangement}

The conducting fluid used was mercury of purity $99.99 \%$, whose density, electrical conductivity and kinematic viscosity were assumed to be $\rho=13.55 \times 10^{3} \mathrm{~kg} \mathrm{~m}^{-3}, \sigma=$ $1.04 \times 10^{6} \Omega^{-1} \mathrm{~m}^{-1}$ and $\nu=1.14 \times 10^{-7} \mathrm{~m}^{2} \mathrm{~s}^{-1}$, respectively. A toroidal configuration was adopted to avoid entrance effects in the duct and to be able to accommodate the device into a solenoidal magnet (figure 1). The cell had a mean radius $r=4.5 \mathrm{~cm}$, with a square cross section with side length $d=1 \mathrm{~cm}$. The walls parallel to the axis of the torus were made of high conductivity copper and were gold plated to minimise the contact resistance by the formation of an amalgam with the mercury. The other two walls (Hartmann walls), along which the Hartmann layers developed (figure 2), were required to be electrical insulators but good thermal conductors, to facilitate the removal of the heat generated by viscous and Joule dissipation in the Hartmann layers. In this way, and given that no external pressure gradient is imposed, the electric currents will be restricted to the Hartmann layers and the mean velocity will depend linearly on their intensity.

The thickness of the Hartmann layers scales like $B^{-1}$, being approximately $3 \mu \mathrm{m}$ for 13 Tesla, the maximum field reached. To determine the influence of the smoothness of the Hartmann walls on the transition process, two different materials were used for these plates. One pair of plates was made of machine-polished painted copper, and their electrical insulation was monitored with wires in contact with the copper. The other plates 
were made of an aluminium nitride ceramic called Shapal, with high thermal conductivity $\left(90 \mathrm{~W} \mathrm{~m}^{-1} \mathrm{~K}^{-1}\right)$ and volume resistivity $\left(10^{14} \Omega \mathrm{cm}\right)$, which was also machine polished. Analyses of the roughness of the plates were carried out at six different locations on their surface. Each sample covered an area of approximately $0.7 \mathrm{~mm}$ by $0.5 \mathrm{~mm}$ and typical profiles are shown in figure 3. The roughness elements in the painted plates were found to have an rms height of $0.1 \mu \mathrm{m}$, with a maximum peak to trough distance of $2 \mu \mathrm{m}$. On the other hand, for the ceramic plates the rms roughness height was $0.6 \mu m$ with maxima of up to $9 \mu m$. Moreover, it can be observed in figure 3 that the variations over the ceramic plates cover a wider range of wavelengths which gives them a higher chance of exciting one or more of the instability modes of the flow. The heat transmitted across the non-conducting walls was removed using water flowing through chambers at the top and bottom of the cell. The temperature of the water flowing through the device was measured using four K-type thermocouples located at the inlets and outlets. It was found that the increase of temperature was less than $4{ }^{\circ} \mathrm{C}$, hence we do not expect substantial variations with temperature in the physical properties of the mercury. Two $3 \mathrm{~mm}$ inner diameter stainless-steel tubes were connected at each side of the internal cavity and were used for filling the device with mercury. A silicon-diaphragm pressure transducer was set connecting the two tubes to measure the radial pressure gradient in the mercury. Assuming that the flow is purely azimuthal the mean velocity, $v_{m}$, was obtained from the balance of momentum in the radial direction given by

$$
\frac{v_{m}^{2}}{r}=\frac{1}{\rho} \frac{\Delta p}{d}
$$

where $\Delta p$ is the pressure difference measured by the transducer across the width of the mercury chamber.

For a fixed magnetic field $B$, an electric current of intensity $I$ was injected between the two conducting walls to drive the mercury. The resulting potential difference $V$ together 
with the readings from the pressure transducer, the thermocouples and the shunt of the power supply were recorded simultaneously with a data acquisition card connected to a PC. Data were obtained at rate of 100 samples per second with 16 bit resolution. From the measurements of the voltage, $V$, and Ohm's law:

$$
J=\sigma\left(-\frac{V}{d}+v B\right)
$$

with $J$ the current density and $v$ the azimuthal component of the velocity, the mean velocity in the flow $v_{m}$ was determined from the integral of (2.2) over the cross-section of the mercury chamber (Baylis 1971):

$$
v_{m}=\frac{V}{d B}-\frac{I}{2 \pi \sigma d^{2} B} \ln \left(\frac{2 r+d}{2 r-d}\right) .
$$

No potential probes were placed in the non-conducting plates to avoid introducing disturbances in the Hartmann layers. Given that there is no externally imposed pressure gradient, the friction factor was defined as

$$
F=\frac{I B}{v_{m}^{2} \rho 2 \pi r}
$$

As mentioned above, from previous experiments on laminarisation we expect the relevant parameter describing the transition to turbulence to be the ratio of the Reynolds number to the Hartmann number, $R=R e / H a$, where

$$
H a=d B \sqrt{\sigma / \rho \nu}, \quad R e=\frac{d v_{m}}{\nu} .
$$

\section{Results and analysis}

The experiments were carried out at the Grenoble High Magnetic Fields Laboratory in France. We used a 10MW resistive magnet which provided a highly homogeneous magnetic field in a cylindrical volume of $13 \mathrm{~cm}$ in diameter for intensities of up to 13 
Tesla. For a given value of $B$, measurements were taken for different intensities of the injected current, which was varied both continuously and at discrete intervals.

We report in first place the results obtained when the painted plates were used as insulating walls. In figure 4 we plotted the values of the friction factor as a function of $R$, calculated with the data obtained from the potential probe and (2.3), for magnetic fields between 1 and 12 Tesla and currents between 0 and 400 Ampère. This corresponds to Hartmann numbers between 130 and 1690, which can be considered well in the high $\mathrm{Ha}$ regime, and to laminar Hartmann layers with thicknesses between approximately 80 and $3 \mu \mathrm{m}$. In the turbulent regime, Tennekes (1966) showed that the displacement thickness of the Hartmann layers $\delta_{t}$, nondimensionalised with respect to its value in the laminar regime, satisfies

$$
\delta_{t}=\frac{R F}{2}
$$

From the results in figure 4 it can be seen that $\delta_{t}$ is only a few times the laminar boundary layer thickness and consequently there is no overlapping between the Hartmann layers at the top and bottom walls of the experimental cell, for the range of Reynolds numbers studied. These measurements were made for values of the electric current spaced by 10 Ampères and changed at intervals of approximately 20 seconds. The results recorded corresponded to the average during the final 10 seconds, that is they comprised 1000 samples. The straight line with slope $-2 / R$ in the plot corresponds to the value of $F$ in the laminar case (Moreau 1990). It can be seen that the experimental results follow this curve well up to $R \approx 380$, where a marked transition occurs and the friction factor takes on higher values than in the laminar case. The critical Reynolds number was found to be the same when the current was decreased in a similar way and the flow was laminarised, i.e. no hysteresis was observed. There is a small displacement between the experimental results and the theoretical curve. As this was found to be the same for the different 
insulating plates and when using different power supplies, we believe it originates in impurities in the mercury or uncertainties in the value of the magnetic field, as read from the controls of the magnet. We found that small variations in any of these factors can make the results coincide with the theoretical curve for the linear regime, without introducing important changes to the characteristics of the transition.

Figure 5 is equivalent to figure 4 but in this case the current was (a) increased and (b) decreased continuously at a rate of $2 \mathrm{~A}$ per second from 0 up to a maximum of $650 \mathrm{~A}$, and all the samples were recorded. It was hoped that the slower variation of the current would help detect any hysteresis. In this case the measurements of $V$ fell in the range 0.1-1.1 Volt and were filtered using a moving average with a window size of 50 samples; the standard deviation was found to be $O\left(10^{-4}\right)$. The behaviour of the friction factor in this case is the same as in figure 4 except for the appearance of important differences in $F$ at low values of the current, between the increasing and decreasing cases, especially at low magnetic fields. From (2.3) and (2.4) it is found that when a variation $\Delta I$ is introduced in the current, the friction factor varies by an amount $\Delta F$, with $\Delta F / \Delta I$ behaving as $I^{-2}$. Given that the temporal rate of variation of $I$ was kept constant, the temporal rate of variation of $F$ become larger the lower the current. Also, the typical time-scale of the damping of vorticity in the flow by viscous stresses in the Hartmann layer (Hartmann friction time) behaves like $B^{-1}$ (Sommeria \& Moreau 1982), meaning that at low $I$ and $B$ the variations in $F$ are more intense and can persist for a period of time that is of the order or longer than the time-window used in filtering the data, resulting in an average that does not correspond to a quasi-steady state of the flow. Measurements were also made varying the current at a lower rate $(1 \mathrm{~A}$ and $0.5 \mathrm{~A}$ per second) and the departures in $F$ were found to diminish. Nevertheless, in all the cases these fluctuations do not affect the flow at values of $R$ around which transition was observed. 
The values of $H a$ and $R e$ covered in figure 5 are compared in figure 6 with those in previous works on laminarisation. It can be seen that in this work we have covered a substantially wider range of $R e$ at higher values of $H a$. The dashed line in the graph corresponds to $R e / H a=250$, which was the highest critical value obtained in the previous laminarisation experiments, and all the results to the right of this curve correspond to turbulent Hartmann layers. The region above the dotted line corresponds to the case when the layers do not overlap according to the model by Alboussière \& Lingwood (2000), thus only the region enclosed by the two curves corresponds to turbulent independent Hartmann layers. It can be seen that this region of parameter space was not extensively explored in the previous experiments.

Although the transition in figure 5 is well marked small variations can be noticed near the top and bottom of the range of $B$. In those cases we believe additional factors introduced instability mechanisms with critical Reynolds numbers commensurate with that for the Hartmann layer. By looking more in detail at the transition region it can be seen in figure 7 (a) that at low magnetic fields, apart from the variations in $F$ due to the longer relaxation time as described above, the laminar regime of the Hartmann layer is not approached until $B \approx 2 \mathrm{~T}$. Given the design of the experimental device, rotational effects need to be taken into account especially at low $B$, when the Lorentz forces can be small compared to inertial effects. In that case it is possible to make a comparison with the flow in curved pipes, where the imbalance near the solid boundaries between the radial pressure gradient and the centrifugal forces induces a secondary poloidal motion, which alters the basic velocity profile and gives rise to additional instability modes. In MHD flows, the damping of the component of the vorticity perpendicular to the magnetic field can reduce significantly the importance of the secondary flow. If the magnetic field is intense enough the flow will be almost purely azimuthal and dominated by the Hartmann 
layers. Baylis \& Hunt (1971) found that the radial and axial components of the velocity can be neglected in comparison to the azimuthal component when

$$
\vartheta=\left(\frac{K}{H a^{2}}\right)^{2} \frac{d}{r} \ll 1,
$$

where $K$ is the Dean number

$$
K=\left(\frac{d}{r}\right)^{\frac{1}{2}} R e .
$$

Baylis (1971) used a set-up similar to ours and followed the gradual transition from a Hartmann regime to one dominated by the secondary flow, and the subsequent abrupt transition to a turbulent state, as the mean velocity was increased. But because the strength of his magnetic field was limited to $0.4 \mathrm{~T}$, he could not observe the direct transition to turbulence in the Hartmann layers. In figure 8 we plotted the values of $\vartheta$ for our measurements at different values of the magnetic field. It can be seen that $\vartheta$ could reach large values at low $R$, especially for $B<1$, and in those cases secondary flow was expected to develop and the transition to be triggered by instability mechanisms associated with rotation. If we assume that the flow near the Hartmann walls can be locally described by a combination of the Hartmann and the Bödewadt layers (the latter corresponding to a flow rotating with constant angular velocity $\Omega$ away from an infinite solid boundary), then the linear stability analysis predicts a critical $R$ below 400 for $B<2 \mathrm{~T}$ (Moresco \& Alboussière 2002a). This instability originates from a combination of viscous effects and streamline curvature and the critical $R$ is a function of the Elsasser number $\Lambda=B^{2} \sigma / 2 \Omega \rho$, that is of the intensity of the magnetic field. We think this dependence gives another estimate for a threshold in $B$ below which rotational effects become important.

In figure 7 (b) is shown a detail of the transition region for $B \geq 4 \mathrm{~T}$. Now the curves corresponding to $4 \leq B \leq 6$ are found to be bundled together in the laminar regime and 
to present approximately the same critical $R$ of 380 . At higher $B$ the critical $R$ decreases with the intensity of the field and the transition becomes less marked. We believe this is due to the increasing importance of the roughness of the Hartmann walls, as the height of the irregularities relative to the boundary layer thickness is proportional to $B$. As pointed out by von Doenhoff \& Braslow (1961), the relevant parameter for the onset of transition is not the rms value of the surface roughness but the maximum projections of individual roughness elements. In table 1 are shown the values of the ratio $\left(\delta_{k}\right)$ of the maximum roughness height to the boundary layer thickness for the two types of insulating plates and different intensities of the magnetic field. It can be seen that in the case of the painted plates this ratio is always less than one, meaning that the highest roughness elements remain submerged inside the Hartmann layer for all the values of $B$ considered. Nevertheless, in more widely studied boundary layers, it has been found that roughness can affect transition even for $\delta_{k}<1$ (Saric, Reed \& Kerschen 2002). For the boundary layers over a swept wing or a flat plate it is known that roughness does not affect transition when the roughness elements are submerged within the viscous sublayer. In that case its effect is limited to an enhancement of the receptivity of free-stream disturbances (c.f. Goldstein 1985; Ruban 1985). For higher values of $\delta_{k}$, the roughness elements in the walls can induce flow separation and the appearance of inflection points which can lead to Rayleigh instability, as shown by Smith \& Bodonyi (1985) for the case of triple deck boundary layers. Although the internal structure of the Hartmann layer is not known we believe the same mechanisms are at work here. The dependence of the critical $R$ on $B$ found in figure 5 and the values in table 1 seem to indicate that when $\delta_{k}>0.3$ roughness effects become important.

Values of the friction factor were also obtained from the measurements of the pressure transducer and are shown in figure 9 for intermediate values of the magnetic field. These 
results coincide with those from the potential probe, although the latter show a lower level of noise and were chosen to be reported here. Nevertheless, the information from the transducer gives an independent confirmation of these results.

The measurements described above were repeated using the ceramic plates as nonconducting walls. As described in $\S 2$, the roughness of those plates is more pronounced than in the painted plates. It can be seen in figure 10 that substantial differences appear between the two sets of results, with the values of the friction factor in the turbulent regime now falling into separate curves for different values of the magnetic field. The critical $R$ for the ceramic plates is found to decrease as the intensity of the magnetic field is increased and the departure from the laminar state becomes more smooth, creating a larger transition region before the flow becomes fully turbulent. The same results were obtained when the intensity of the current was increased or decreased, and when it was varied continuously or by discrete intervals. This behaviour resembles that found for the painted plates for $B \geq 7$ although in a much more marked way. In that case it was found that roughness affects transition when $\delta_{k}>0.3$. For the ceramic plates this was the case for $B>1$ (see table 1), that is, for most of the data in figure 10, given further support to the statement that 0.3 is approximately a cut-off value for $\delta_{k}$ above which roughness becomes important. The same influence of roughness on transition was found by Dukure (1965) in experiments of magnetohydrodynamic channel flow with considerably higher roughness, reporting critical values of $R$ as low as 30 for $\delta_{k} \approx 6$. Our results also show that in the turbulent regime, the friction factor becomes increasingly independent of $R$ as the magnetic field is increased, being a function only of the ratio of the roughness to boundary layer thickness, i.e. of $B$, a behaviour characteristic of the rough turbulence regime. Although roughness was found to have a marked effect on transition and the turbulent regime, it does not seem to affect the values of the friction factor in the lami- 
nar Hartmann layer, even when the height of the roughness elements is several times the boundary layer thickness.

\section{Conclusions}

The transition to turbulence in the Hartmann layer in the high $\mathrm{Ha}$ regime was investigated through measurements of the friction factor in the electromagnetic flow in a square duct. The walls parallel and perpendicular to the magnetic field were good electrical conductors and insulators respectively, and the flow was driven by the interaction of an externally imposed magnetic field and an injected current. The transition process was studied in detail by using slow variations of the electric current (velocity) so as to detect any hysteresis between transition and laminarisation. The critical value of the Reynolds over Hartmann number $(R)$, was found in both cases to be the same and approximately equal to 380 . The dependence of the critical $R$ on the roughness characteristics of the Hartmann walls confirms that the transition observed is highly related to changes in the stability of these layers. The critical value of $R$ obtained is higher than in previous experiments on laminarisation, which locate it in the range $150<R<250$. Also the characteristics of the transition appear to be different as it can be seen in figure 11, where only the previous laminarisation data for nonoverlapping Hartmann layers were plotted. While in the laminarisation studies the critical $R$ was identified with a smooth departure of the friction factor from its value in the laminar regime, in our experimental results a very well marked transition is observed. One possible reason for this difference is the substantially higher value of the Hartmann number reached in this work, as a tendency to increasing critical $R$ with $H a$ can also be observed in the previous works (see figures 6 and 8 of Alboussière \& Lingwood (2000)). It also has to be taken into account that using a different forcing mechanism, electromagnetic forces in this case and pressure 
gradients in the previous studies, can have effects in the global stability properties, as the electric current distributions in both cases are different.

Like in the experiments on laminarisation, the critical value of $R$ obtained here is two orders of magnitude smaller than that predicted by the linear stability theory. It seems nonlinear effects can be triggered at subcritical values of $R$ by small but finite size disturbances, which could originate in the parallel layers, given that previous works suggest they present a substantially lower critical Reynolds number. The interaction between the parallel and Hartmann layers, especially in the corner regions of the ducts, would require an stability analysis where both types of layers are considered simultaneously. The experimental results presented here together with numerical evidence of the existence of transient growth of disturbances at low $R$ (Gerard-Varet 2002), suggest that the critical value of $R$ is very sensitive to the magnitude of the disturbances. This seems to be confirmed by results from direct numerical simulations by Andreev et al. (2002), who showed that the Hartmann layer can sustain turbulence for values of $R$ close to 400 when excited with suitable chosen three dimensional disturbances, and is also in agreement with the energetic stability analysis of Lingwood \& Alboussière (1999), which yielded a critical $R \approx 26$ for global stability, i.e. the limit below which no transfer of energy is possible between a disturbance, independently of its size, and the basic flow.

This work was supported in part by the Engineering and Physical Sciences Research Council of the UK, grant GR/N04072.

\section{REFERENCES}

Alboussière, T. \& Lingwood, R. J. 2000 A model for the turbulent Hartmann layer. Phys. Fluids 12, 1535-1543.

Andreev, O., Kolesnikov, Y., Krasnov, D., Roth, E., Thess, A. \& Zienicke, E. 2002 
Experimental and numerical study of elecrtromagnetic braking in liquid metals. In Proc. Fifth Internat. Pamir Conf., Ramatuelle, France.

BAYLIS, J. A. 1971 Experiments on laminar flow in curved channels of square section. J. Fluid Mech. 48, 417-422.

BAYlis, J. A. \& Hunt, J. C. R. 1971 MHD flow in an annular channel; theory and experiment. J. Fluid Mech. 48, 423-428.

Branover, G. G. 1967 Resistance of magnetohydrodynamic channels. Magnetohydrodynamics 3, 1-11.

Burr, U., Barleon, L., Müller, U. \& Tsinober, A. 2000 Turbulent transport of momentum and heat in magnetohydrodynamic rectangular duct flow with strong sidewall jets. J. Fluid Mech. 406, 247-279.

von Doenhoff, A. E. \& Braslow, A. L. 1961 The effect of distributed surface roughness on laminar flow. In Boundary layer control, pp. 657-681. Pergamon.

DukURE, P. K. 1965 Effect of relative roughness on a conducting fluid flow in the presence of a magnetic field. Magnetohydrodynamics 1, 20-22.

Garandet, J. P. \& Alboussière, T. 1999 Bridgman growth: modelling and experiments. Prog. Crystal Growth and Charact. 38, 73-132.

Gerard-Varet, D. 2002 Amplification of small perturbations in a Hartmann layer. Phys. Fluids 14, 1458-1467.

Goldstein, M. E. 1985 Scattering of acoustic waves into Tollmien-Schlichting waves by small streamwise variations in surface geometry. J. Fluid Mech. 154, 509-529.

HaRTmann, J. 1937 Theory of the laminar flow of an electrically conductive liquid in a homogeneous magnetic field. K. Dan. Vidensk. Selsk. Mat. Fys. Medd. 15(6), 1-28.

Hartmann, J. \& Lazarus, F. 1937 Experimental investigations on the flow of mercury in a homogeneous magnetic field. K. Dan. Vidensk. Selsk. Mat. Fys. Medd. 15(7), 1-45.

Hunt, J. C. R. 1965 Magnetohydrodynamic flow in rectangular ducts. J. Fluid Mech. 21, 577-590.

Lingwood, R. J. \& Alboussière, T. 1999 On the stability of the Hartmann layer. Phys. Fluids 8, 2058-2068. 
LOCK, R. C. 1955 The stability of the flow of an electrically conducting fluid between parallel planes under a transverse magnetic field. Proc. R. Soc. Lond. A. 233, 105-125.

LykOUdis, P. S. 1960 Transition from laminar to turbulent flow in magneto-fluid mechanic channels. Rev. Mod. Phys. 32, 796-798.

Moreau, R. 1990 Magnetohydrodynamics. Kluwer.

Moresco, P. \& Alboussit̀re, T. $2002 a$ Stability of Bödewadt-Hartmann layers. Submitted to Phys. Fluids.

Moresco, P. \& Alboussière, T. $2002 b$ Weakly nonlinear stability of Hartmann boundary layers. To appear in Eur. J. Mech., B/Fluids.

Murgatroyd, W. 1953 Experiments on magneto-hydrodynamic channel flow. Philos. Mag. 44, $1348-1354$.

Reed, C. B. \& Picologlou, B. F. 1989 Sidewall flow instabilities in liquid metal MHD flow under blanket relevant conditions. Fusion Technol. 15, 705-715.

Ruban, A. I. 1985 On the generation of Tollmien-Schlichting waves by sound. Fluid dyn. 19, $709-716$.

SARIC, W. S., REED, H. L. \& KERSChEn, E. J. 2002 Boundary-layer receptivity to freestream disturbances. Ann. Rev. Fluid Mech. 34, 291-319.

Schlichting, H. 1960 Boundary Layer Theory. McGraw-Hill Book Company,USA.

Smith, F. T. \& Bodonyi, R. J. 1985 On short-scale inviscid instabilities in flow past surfacemounted obstacles and other non-parallel motion. Aero. J. Paper 1313 pp. 205-212.

Sommeria, J. \& Moreau, R. 1982 Why, how, and when, MHD turbulence becomes twodimensional. J. Fluid Mech. 118, 507-518.

Tennekes, H. 1966 Turbulent magnetohydrodynamic channel flow. Phys. Fluids 9, 1876-1879.

Ting, A. L. \& WALKeR, J. S. 1991 Linear stability analysis for high-velocity boundary layers in liquid-metal magnetohydrodynamic flows. Int. J. Engng Sci. 29, 939-948. 


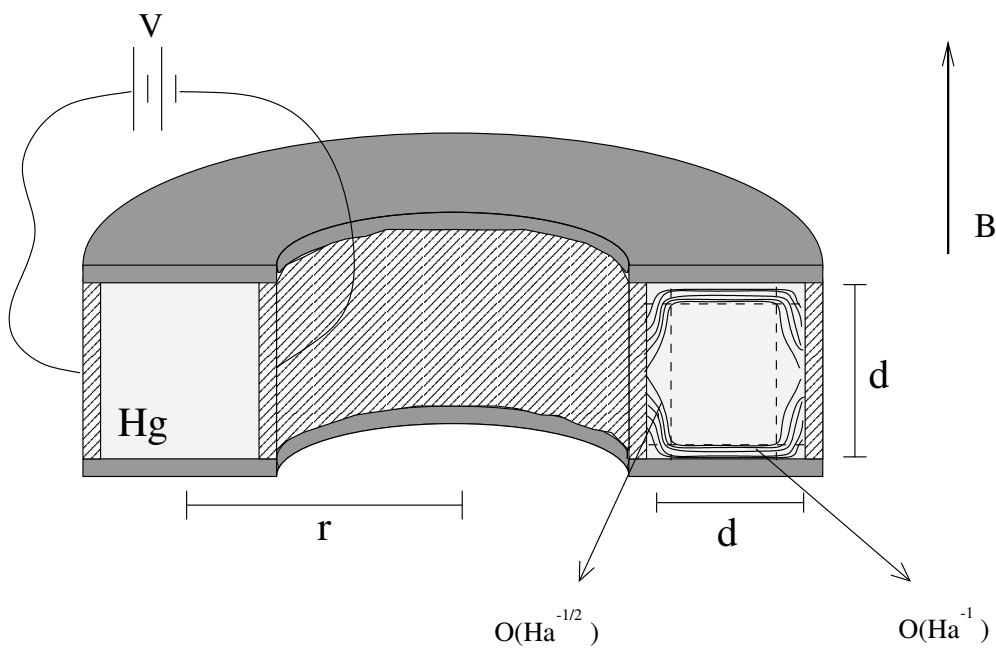

Figure 1. Scheme of the experimental device. 


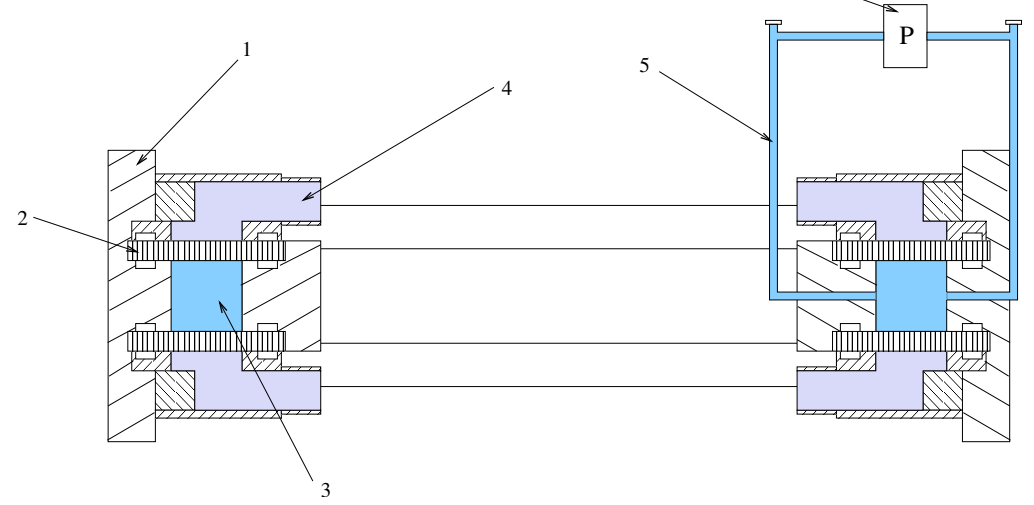

FIGURE 2. Scheme of the cross section of the experimental device: 1. High conductivity copper. 2. Nonconducting (Hartmann) walls. 3. Mercury chamber. 4. Cooling water chambers. 5. Stainless steel tubes. 6. Pressure transducer. 


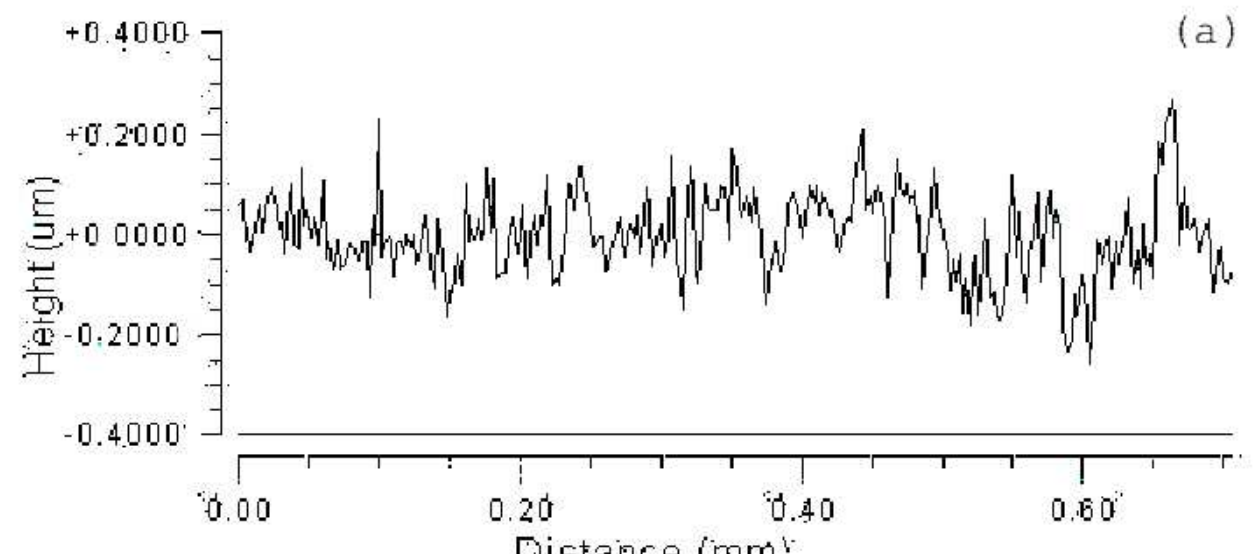

Distance ( $\mathrm{mm}$ )

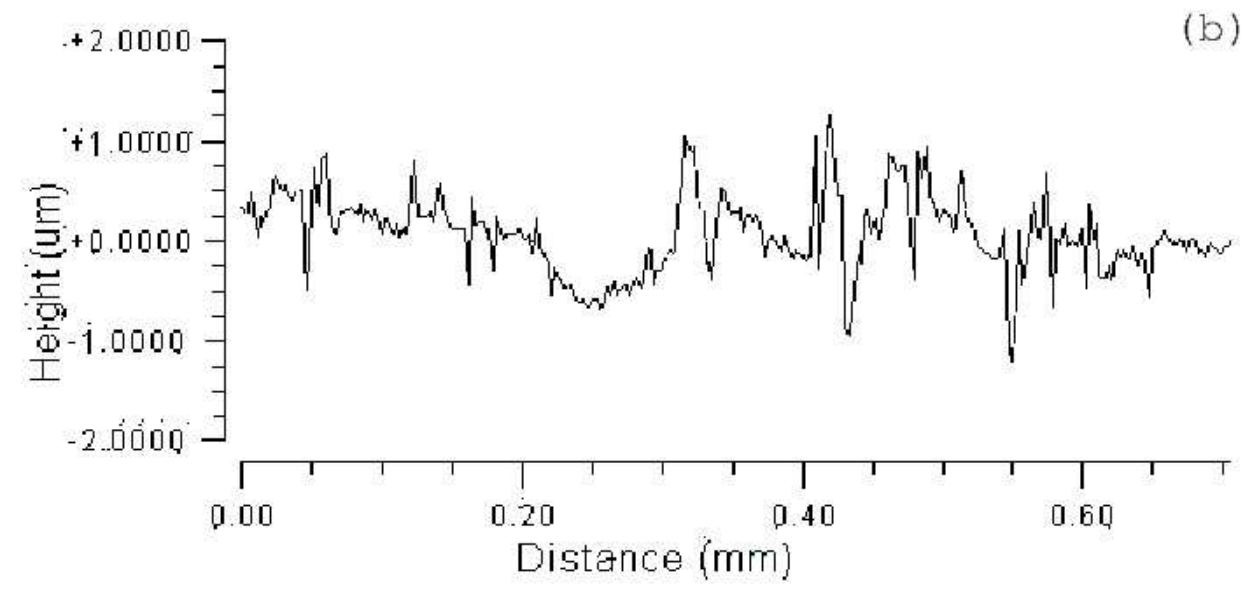

Figure 3. Roughness profiles of the (a) painted and (b) ceramic plates. 


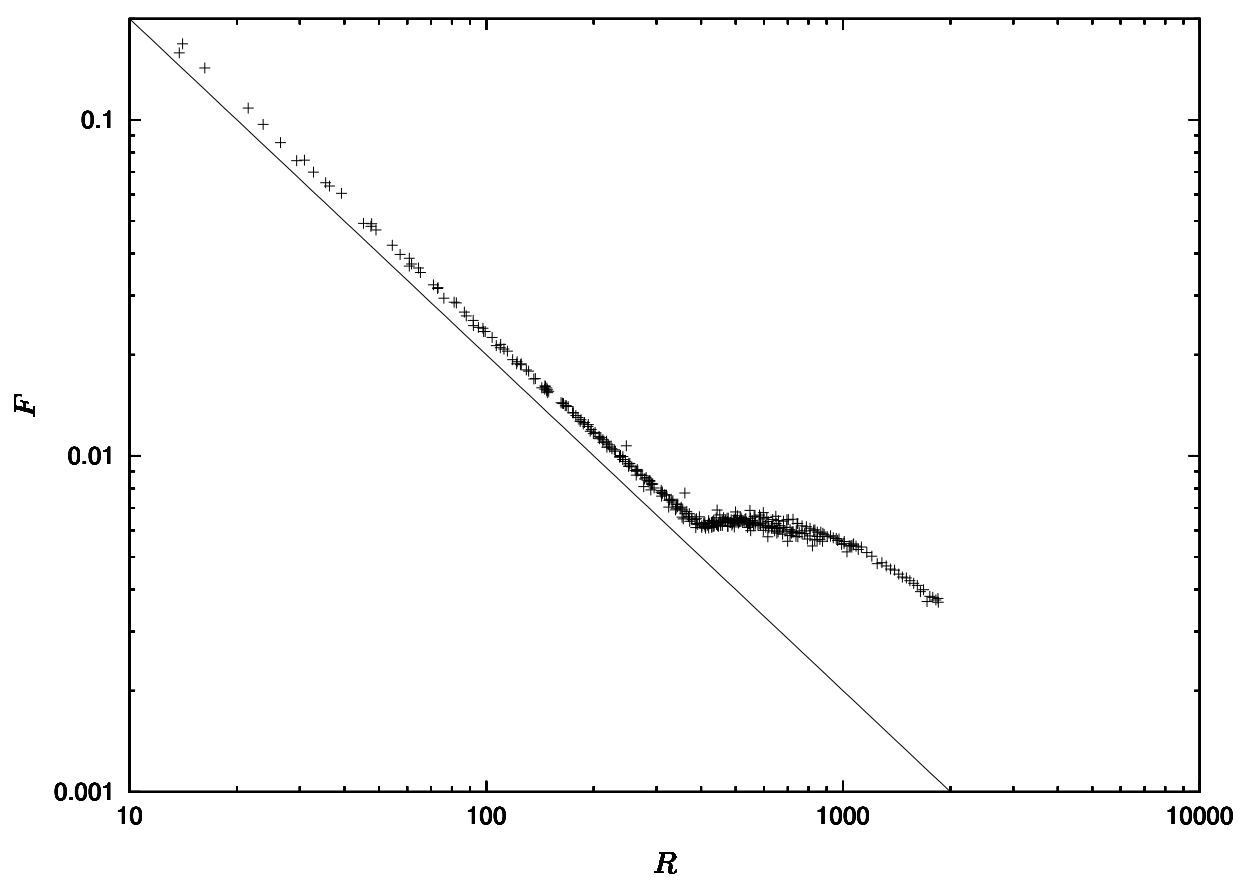

FIgURE 4. Friction factor for discrete values of the current between 0 and $400 \mathrm{~A}$, and magnetic fields between 1 and $12 \mathrm{~T}$. The nonconducting walls were painted copper plates. The straight line corresponds to the friction factor for laminar Hartmann layers. 

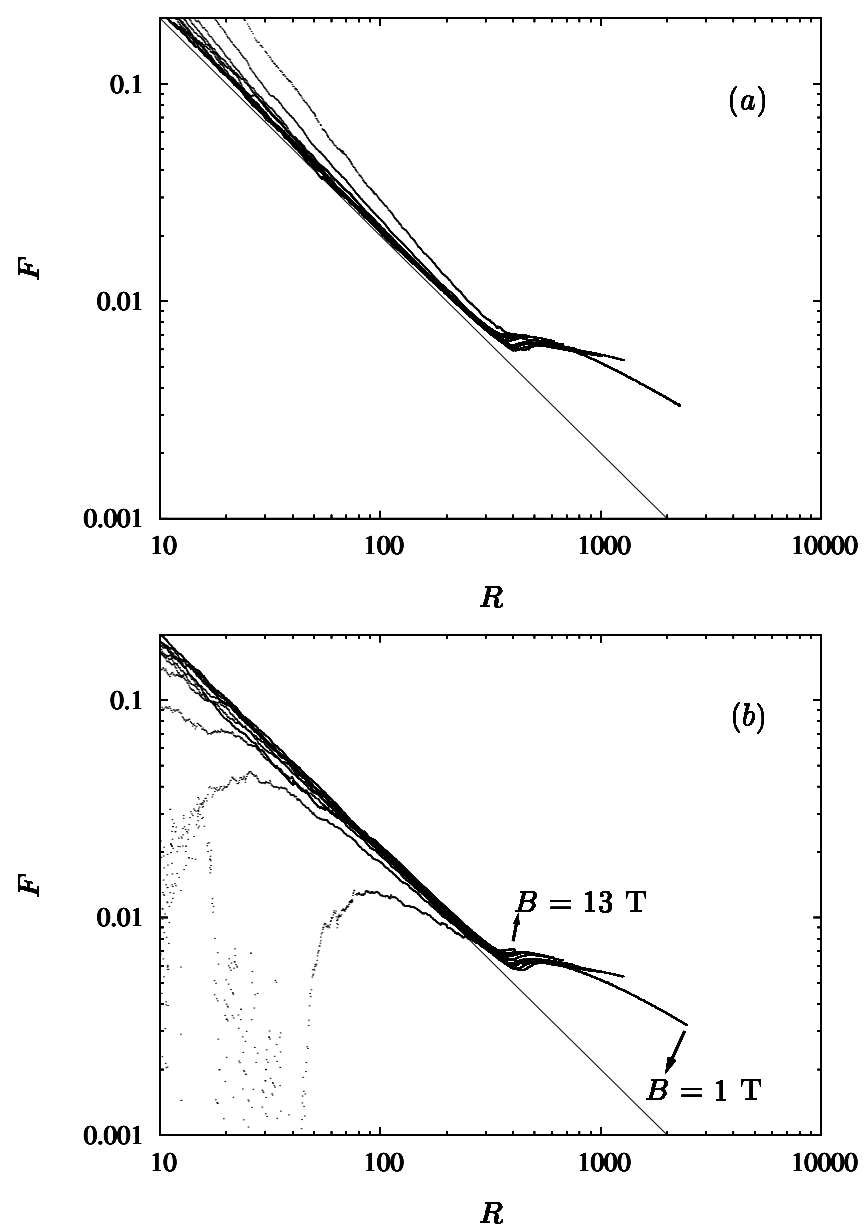

FIgURE 5. Friction factor for continuous values of increasing (a) and decreasing (b) electric current between 0 and $650 \mathrm{~A}$, and magnetic fields between 1 and $13 \mathrm{~T}$. The nonconducting walls were painted copper plates. The straight line corresponds to the friction factor for laminar Hartmann layers. 


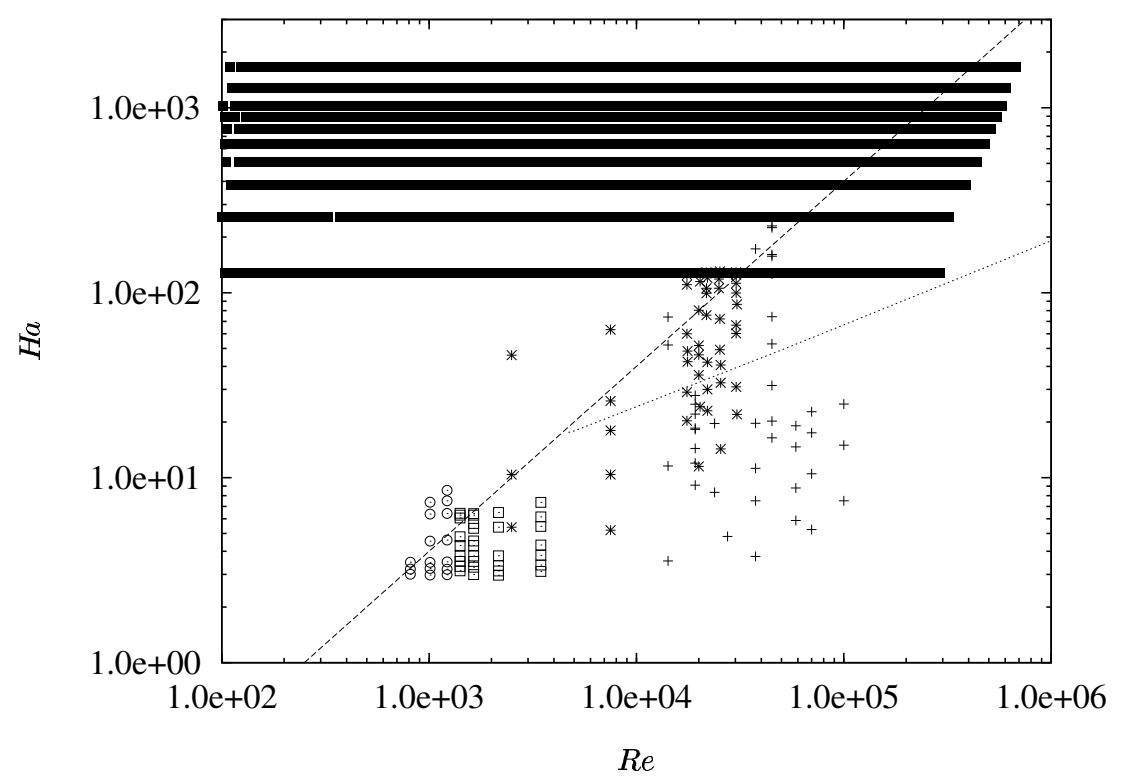

FIgURE 6. Values of the Reynolds and Hartmann numbers corresponding to the experimental results in figure 5 (ם). Previous works: $\square$ Branover (1967), $\odot$ Hartmann \& Lazarus (1937), * Murgatroyd (1953), + Lykoudis (1960). The dashed line corresponds to Re/Ha=250 and the dotted line marks the limit below which the Hartmann layers overlap. 

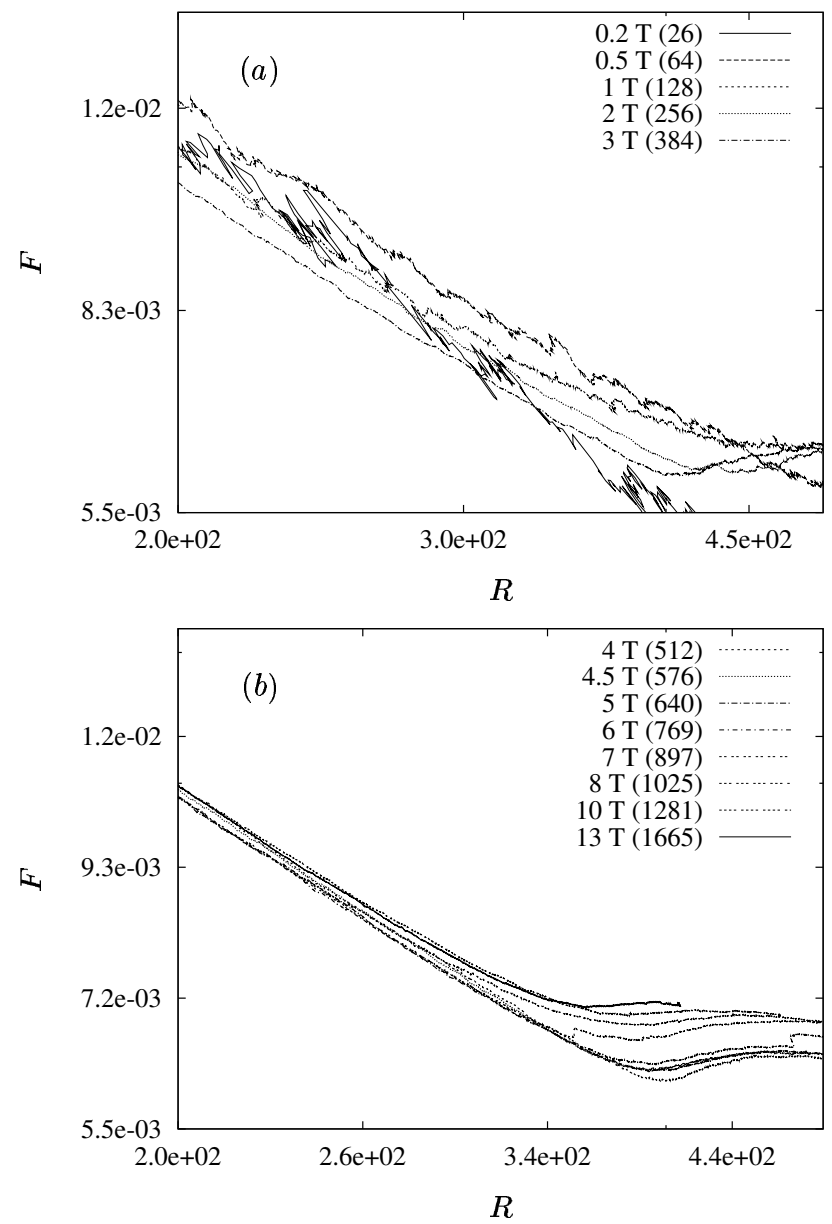

FIGURE 7. Friction factor near the transition region for the same conditions as in figure 5 at (a) low and (b) high magnetic fields. The corresponding Hartmann number is indicated between brackets. 


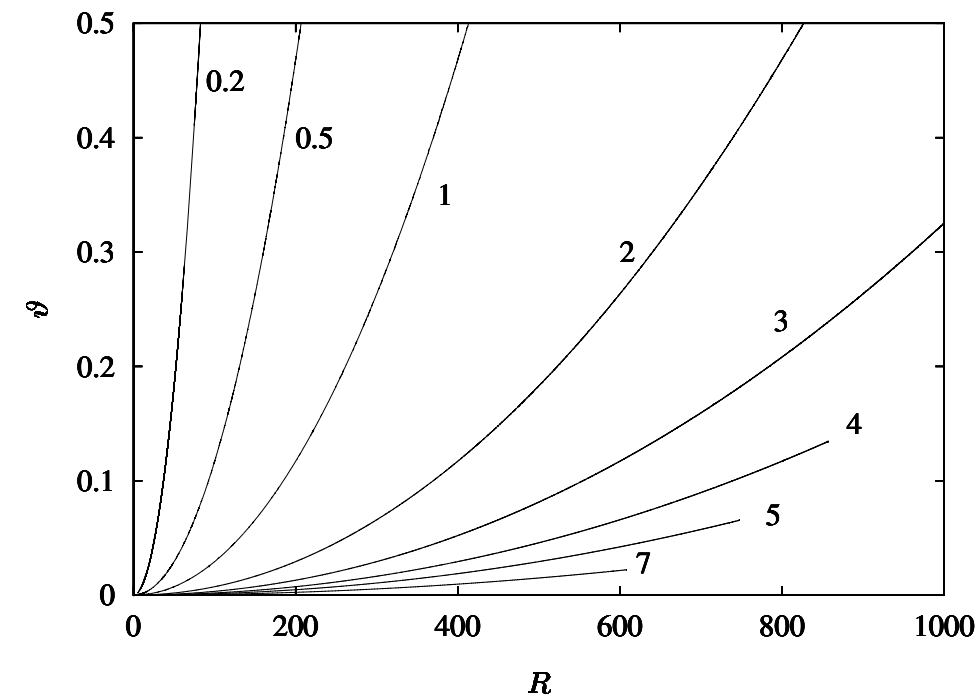

FIGURE 8. Intensity of the secondary flow in the experimental data as measured by the parameter $\vartheta$. The value of the magnetic field in Tesla is indicated next to each curve. 


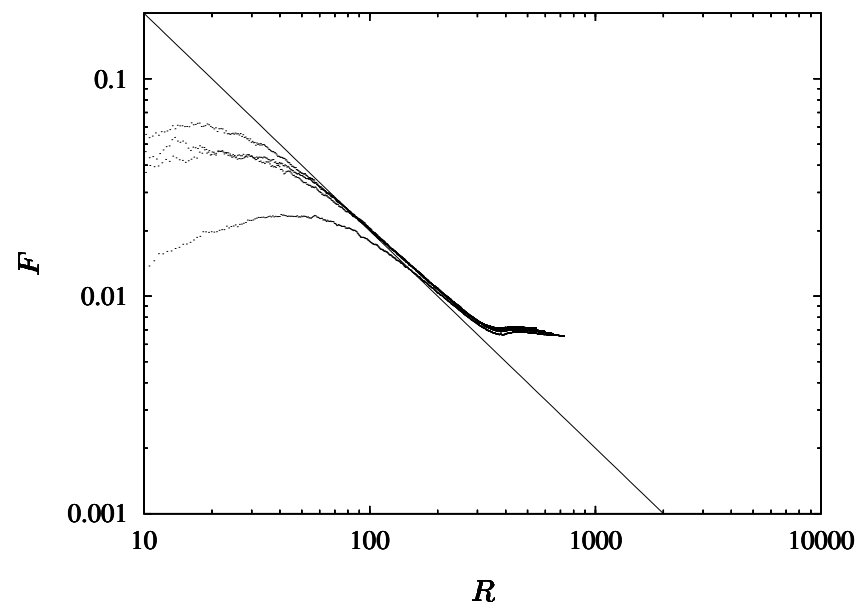

FIGURE 9. Friction factor using the painted plates obtained with the readings from the pressure transducer for fields between 4 and $8 \mathrm{~T}$ and decreasing currents. The straight line corresponds to the friction factor for laminar Hartmann layers. 

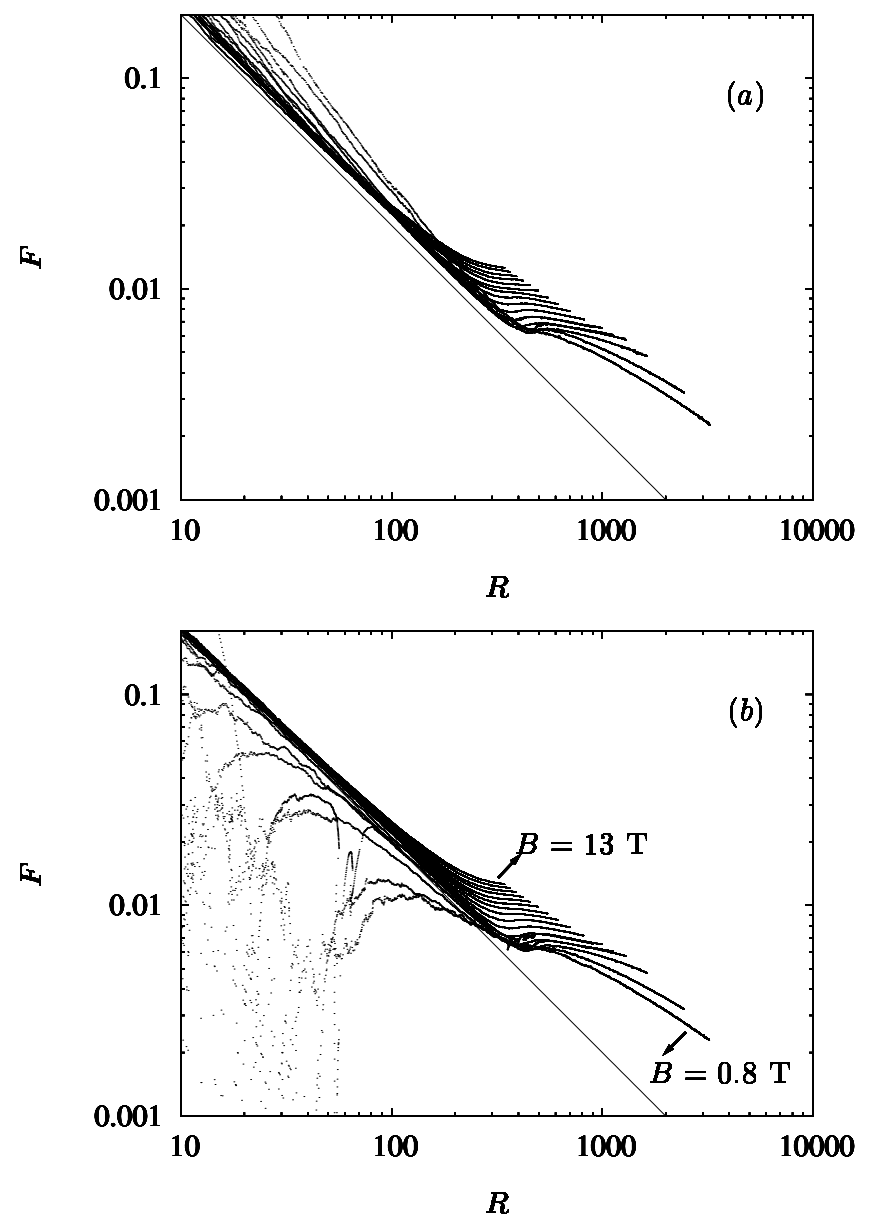

FIgURE 10. Friction factor for the ceramic plates for continuous values of increasing (a) and decreasing (b) electric current between 0 and $650 \mathrm{~A}$, and magnetic fields between $0.8(\mathrm{Ha}=102)$ and $13(H a=1665)$ Tesla. The straight line corresponds to the friction factor for laminar Hartmann layers. 


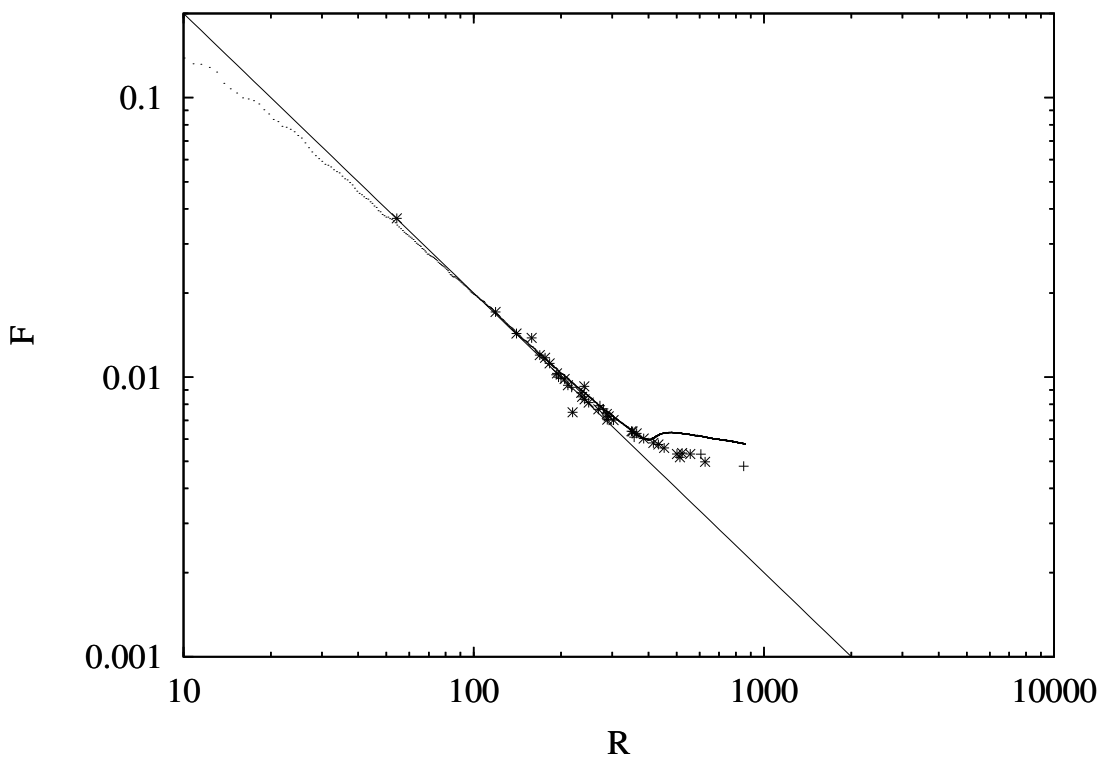

FIguRE 11. Comparison of the data in figure 5 for a magnetic field of 4 Tesla $(H a=512)$ with the results on laminarisation from nonoverlapping Hartmann layers (see figure 6 for key to symbols). 


$\begin{array}{lcc}B & \text { Painted } & \text { Ceramic } \\ 1 & 0.04 & 0.19 \\ 2 & 0.10 & 0.47 \\ 3 & 0.16 & 0.70 \\ 4 & 0.21 & 0.94 \\ 5 & 0.26 & 1.17 \\ 6 & 0.31 & 1.4 \\ 7 & 0.36 & 1.64 \\ 8 & 0.42 & 1.88 \\ 9 & 0.47 & 2.11 \\ 10 & 0.52 & 2.34 \\ 11 & 0.57 & 2.58 \\ 12 & 0.62 & 2.81 \\ 13 & 0.68 & 3.04\end{array}$

TABLE 1. Ratio of the maximum roughness height to Hartmann layer thickness for different values of the magnetic field $B$ in Tesla and the two types of Hartmann walls. 